\title{
Electron Transport in Magnetic Quantum Point Contacts
}

\author{
T. Pietsch*, S. Egle, C. Espy, F. Strigl and E. Scheer \\ Department of Physics, University of Konstanz, 78457 Konstanz, Germany
}

\begin{abstract}
In recent years, the fabrication of novel building blocks for quantum computation- and spintronics devices gained significant attention. The ultimate goal in terms of miniaturization is the creation of single-atom functional elements. Practically, quantum point contacts are frequently used as model systems to study the fundamental electronic transport properties of such mesoscopic systems. A quantum point contact is characterised by a narrow constriction coupling two larger electron reservoirs. In the absence of a magnetic field, the conductance of these quantum point contacts is quantised in multiples of $2 e^{2} / h$, the so-called conductance quantum $\left(G_{0}\right)$. However, in the presence of magnetic fields the increased spin-degeneracy often gives rise to a deviation from the idealized behaviour and therefore leads to a change in the characteristic conductance of the quantum point contact. Herein, we illustrate the complex magnetotransport characteristics in quantum point contacts and magnetic heterojunctions. The theoretical framework and experimental concepts are discussed briefly together with the experimental results as well as potential applications.
\end{abstract}

PACS: 73.23.-b, 72.25.-b, 73.63.-b

\section{Introduction}

The ongoing trend towards smaller and more efficient electronic devices drives the need for novel, functional building blocks. The ultimate level of miniaturization is achieved when single atoms or molecules are incorporated as functional elements into new types of devices. Besides the technical challenges involved in fabricating such devices, their realization requires both a detailed understanding of the transport properties in confined geometries, which can be significantly different from the respective bulk material, and the ability to control the electronic transport through single atoms and molecules.

In the past, transport spectroscopy has been established as a surprisingly simple technique to investigate the fundamental physical properties of materials in the size range of single atoms to some $100 \mathrm{~nm}$. In essence, a current is driven through a nanometre-sized conductor and its voltage response is monitored as function of external factors, such as the junction diameter, the temperature or the application of a magnetic field. The propagation of electron waves through such a junction reveals information on its chemical nature and electronic structure as well as mechanical properties [1]. This method has been applied to study metallic conductors [2-4], superconductors $[5-8]$ and individual molecules $[9-12]$ as well as carbon nanotubes [13] among others to understand multiple phenomena that can be observed solely at the nanoscale, including conductance quantization, the Coulomb blockade and the Andreev reflection.

\footnotetext{
* corresponding author; e-mail:

torsten.pietsch@uni-konstanz.de
}

The special case of a single atom connecting two larger electrodes is of particular importance because the electronic transport is then dominated by this single atom, giving access to its most fundamental properties. The system is also simple enough to be accurately described by theoretical models, which allows a direct comparison of theory and experiment. In this respect, an interesting question concerns the role of magnetism and the effect of the electron spin on the transport properties of such a contact. Since this issue is also technologically important, especially in the view of novel concepts for data storage [14-17], the study of transport through individual magnetic atoms and molecules has become the subject of intensive studies [15, 18-22]. Despite the immense progress in the field there are numerous open questions, e.g. whether materials that are not known to be ferromagnetic in bulk may become magnetic at the nanoscale or how a spin-polarized current is transported in ferromagnetic single-atom contacts.

This article attempts to give a short introduction into the preparation and characterisation of atomic-sized contacts using the mechanically controllable break-junction technique. In particular, we present recent results on magnetic point contacts and heterocontacts based on $\mathrm{Co}$, $\mathrm{Pt}$ and $\mathrm{Au}$.

\section{Realisation of magnetic quantum point contacts}

Since the early experiments on metallic point contacts by Yanson et al. $[2,23-26]$ in the beginning of the 1970s, a wide variety of methods have been developed to study the ballistic transport in confined geometries and monoatomic junctions. The most common method to create a point contact with diameters of $10 \mathrm{~nm}$ to 
$100 \mathrm{~nm}$ between two metals is known as needle-anvil technique [27], where a metallic needle is gently brought into contact with a metallic film. Typically, the contact resistance ranges from $0.1 \Omega$ to $10 \Omega$ and ballistic transport has been demonstrated in clean metallic contacts at low temperatures. The term ballistic refers to a transport mechanism, in which the electrons travel through a constriction without being scattered by defects or surfaces. Despite the absence of scattering in sufficiently small, ballistic contacts, the electrons experience a quantum resistance, which limits the current. However, due to the lack of stability the needle-anvil technique is not suited to study smaller contacts in the range of few atoms, where the quantum nature of the electron transport becomes dominant.

Such contacts can be achieved using the mechanically controllable break junction (MCBJ) technique [3, 28-32] and STM-based methods [33-36]. The advantages of the MCBJ technique are its high vibration stability and the fact that the junction resistance can be adjusted over a wide range from $10^{-3} \Omega$ to $>10^{4} \Omega$. Moreover, a vacuum tunnelling contact between the electrode tips can be realized and molecules bridging the electrode gap may be deposited to form a single-molecule junction [12, 37-39].

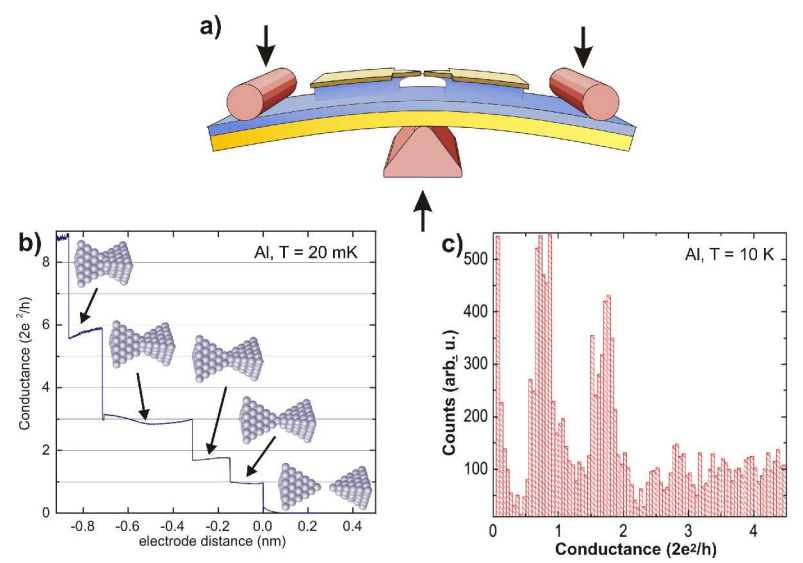

Fig. 1. (a) Schematic illustration of a MCBJ sample mounted in the bending mechanism and typical arrangement of the mechanical setup. (b) Example of an opening trace of an $\mathrm{Al}$ break junction; the insets show schematically the cross-section of the atomic contact. (c) Histogram of an Al break junction obtained via statistical analysis of 33 individual opening traces (courtesy: T. Böhler).

Additionally, upon opening the contact is formed between freshly broken, clean surfaces reducing the probability to accumulate contaminations from the surrounding environment. Hence, ultraclean and stable break junctions can be prepared at cryogenic temperatures in vacuum. Another advantage is that, with microfabricated MCBJs, one can easily create heterocontacts, where the nanogap is formed between two different metals. This is especially important for the investigation of spin-electronic effects, where the resistance of the junc- tion is affected by spin-dependent scattering of electrons at the interface of different magnetic and non-magnetic materials.

A schematic representation of the MCBJ technique is shown in Fig. 1a. A flexible substrate, normally phosphor bronze covered with a thin insulating layer of polyimide (kapton $\left.{ }^{\circledR}\right)$, is mounted in a three-point bending configuration between two fixed counter supports and a movable pushing rod. The substrate is bent by driving the pushing rod vertically towards the substrate using either a piezo-stack or some kind of mechanical gear arrangement (e.g. a differential screw). The metal to be studied is fixed on top of the flexible substrate; this can be done by simply gluing a notched wire $[3,32]$ onto the sample or by using a microfabricated metal film [40, 41]. The vertical motion of the pushing $\operatorname{rod}(\mathrm{d} z)$, which is bending the substrate, leads to a horizontal displacement of the electrodes $(\mathrm{d} u)$. The displacement ratio is approximately given by [42]: $r_{\mathrm{d}}=\frac{\mathrm{d} u}{\mathrm{~d} z}=\frac{6 t u}{2 L}$, where $t$ is the thickness of the sample and $L$ is the separation of the counter supports. Upon stretching, the metallic junction forms a constriction until the nanobridge breaks into two clean surfaces. These surfaces can be brought into contact again by relaxing the force on the pushing rod and thus reforming the contact.

Figure 1b shows conductance traces during the opening and closing of an $\mathrm{Al}$ break-junction. The last conductance plateau before breaking the junction usually corresponds to a single-atom contact, which shows a characteristic conductance value depending on the type of metal under investigation. Normally, such mechanical break junctions can be opened and closed several hundreds of times without permanently destroying the sample.

This excellent stability can be exploited to create a conductance histogram (Fig. 1c) via statistical analysis of many hundreds of opening-closing traces. Individual conductance traces often display a rich variety of features, which partially conceal the statistically preferred conductance values. Therefore, conductance histograms (Fig. 1c) provide an important tool for data analysis. Besides these opening and closing traces, one can access the current-voltage characteristics of the junction as well as the magnetoconductance at a fixed position of the pushing rod; these measurements yield additional information on the band structure of the metal and relevant scattering phenomena in the vicinity of the constriction.

Recently, a number of different methods evolved to fabricate point contacts based on direct nanofabrication techniques. However, these techniques usually yield fixed contacts with diameters ranging from the few- or single atom regime up to about $30 \mathrm{~nm}$ or more. Due to the numerous advantages and the flexibility of the MCBJ technique, herein we will focus on microfabricated MCBJs based on para- and ferromagnetic metals as well as heterojunctions of different metals. 


\section{Electron transport through atomic-sized contacts}

The electrical properties of conventional bulk conductors can be appropriately described by Ohm's law, which states that the conductance only depends on the conductivity of the material and the ratio of cross-section to length of the sample. This simple concept no longer holds true if one considers that a wire of those diameter is in the range of few nanometres.

In a contact between two non-magnetic metal electrodes the conductance becomes quantized when the diameter of the contact approaches the Fermi wavelength of the electrons in the metal, e.g. in MCBJs one observes distinct conductance steps in the order of the conductance quantum $G_{0}=2 e^{2} / h=1 / 12960 \Omega^{-1}$ in the opening traces (Fig. 1b); here $e$ is the elementary charge and $h$ is Planck's constant. The basic idea is that the junction goes through a series of sudden atomic rearrangements as it is stretched, while each atom contributes to the total conductance of the junction by supporting a given number of conductance channels with variable transmission $\tau$. Since only certain contact diameters are allowed due to atomic constraints, the conductance changes in a stepwise fashion as the electrodes are pulled apart.

The total conductance of a given ensemble of conduction channels can be described using the well-known Landauer formula [1]:

$$
G=\frac{2 e^{2}}{h} \sum_{n} \tau_{n},
$$

where $\tau_{n}$ is the transmission coefficient of the $n$-th channel or eigenmode, $e^{2} / h$ is the conductance quantum per electron spin and the factor two accounts for the spin-degeneracy. In a situation, where the transmission of each conductance channel is equal to one, the Landauer formula (1) simplifies to $G=\frac{2 e^{2}}{h} N$, where $N$ is the number of open channels in the contact. In this situation the conductance is quantised to multiples of the conductance quantum $2 e^{2} / h$. Assuming a single conductance channel per atom in the junction, the number of atoms in the cross-section of the constriction is given by $N$.

In a magnetic point contact, however, this simple picture becomes somewhat more complicated because exchange interaction removes the degeneracy of spin-up and spin-down subbands, leading to a pronounced spin-polarization at the Fermi edge. If only one type of electrons (spin-up or spin-down) contributes to the conductance through fully spin-polarized channels, Eq. (1) may be written as [43]:

$$
G=\frac{e^{2}}{h} \sum_{n}\left(\tau_{n \downarrow}+\tau_{n \uparrow}\right),
$$

where $\tau_{\uparrow}$ and $\tau_{\downarrow}$ are the transmission probabilities for the spin-up and spin-down eigenmodes, respectively. If, in addition, only one spin direction had $\tau=1$ while the other ones were blocked, naturally one would assume that half-integer steps of the conductance quantum are ob- served in the opening traces, e.g. $\Delta G=\frac{G_{0}}{2}=\frac{e^{2}}{h}$. Indeed, half-integer conductance quanta have been observed in both magnetic- and non-magnetic point contacts, particularly in external magnetic fields [43-50].

However, it is still highly controversial whether the occurrence of half-integer conductance values can serve as absolute proof for the influence of magnetism on the electron transport in point contacts. For example, there are numerous reports demonstrating the absence of $G_{0} / 2$ conductance steps in magnetic junctions, both experimentally [51, 52] and theoretically [53-59]. Moreover, half-integer conductance quantization has also been found as a result of contaminations of the point contact with $\mathrm{H}_{2}$ and $\mathrm{CO}$ [60]. Therefore, the interpretation of the conductance in magnetic point contacts is not always straightforward.

Frequently, the precise atomic structure at the contact area is unknown, making a detailed modelling of the transport properties almost impossible. In particular the magnetic state of a single atom or monoatomic wire coupled to the leads is not accessible experimentally. Indications of the presence of local magnetic moments in the constriction can be deduced from the appearance of the so-called Kondo effect, distinct nonlinearities around zero-bias in the current-voltage characteristics. By this method evidence for local moments was found for atomic contacts of the three band magnets $\mathrm{Fe}, \mathrm{Ni}$, Co [61].

\section{Ferromagnetic ( $\mathrm{Co}$ ) single-atom contacts}

The properties of magnetic materials play an important role in the design of spin-electronic devices, which rapidly gained technological significance since the independent discovery of the giant magnetoresistance (GMR) effect by Grünberg et al. [62] and Fert et al. [63], for which both were awarded the Nobel prize in 2007.

In its simplest realization a GMR device consists of two ferromagnetic electrodes separated by a normal metal interlayer. The signature of the GMR effect is a differing resistance value (by roughly $10 \%$ to $20 \%$ ) in the parallel and the antiparallel magnetization state of the two electrodes. Shortly after the discovery of the GMR, the tunnel magnetoresistance (TMR) was reported.

TMR is a similar effect as GMR, but the two magnetic electrodes are coupled via a tunnel barrier rather than a normal metal. As a result the MRR values are higher and the typical magnetoresistance (MR) traces slightly differ from those of GMR devices.

Besides these effects, which are restricted to heterostructures, several mechanisms are known to contribute to the MR, i.e. the dependence of the resistance of a system on an external magnetic field. These include the anisotropic magnetoresistance (AMR), which denotes a different resistance value of a magnetic sample depending on the relative orientation of the magnetization and the current directions, and various quantum interference effects. Still, the discovery of the GMR with its relatively high value of several percent came as a surprise because 
the MR in homogeneous three-dimensional systems is in general a small effect amounting to less than $1 \%$.

However, in reduced dimensions magnetoresistance changes may become more pronounced as demonstrated e.g. by the quantum Hall effect in two-dimensional systems showing ballistic electron transport in strong external magnetic fields.

When the dimensions of magnetic materials are reduced to the scale of a single atom, the quasicontinuous electron band structure of the bulk material is successively transformed into the discrete energy spectrum of a single atom. As a result, unexpected magnetic properties can be observed, which result from the interaction and occupation of spin-up and spin-down states. Unlike monovalent metals, such as gold, which exhibit a single conductance channel per atom [30], multivalent transition metals usually support multiple conductance channels.

For a single transition metal atom with partially occupied $d$-states the number of conductance channels has been predicted to be five [1]. Normally, all transmission modes are only partially open $\left(\tau_{n \uparrow}, \tau_{n \downarrow}<1\right.$ in Eq. (2)), giving rise to a total conductance of $1.3 G_{0}$ for a single atom contact. Hence, the magnetic moment of the central atom in the junction and spin-dependent transmission through the given ensemble of channels strongly influences the position of preferred conductance values in the conductance histogram. The understanding of these complex multichannel systems at the atomic scale, in particular the interplay of different spin states, magnetization and quantized conductance, is one of the most fundamental questions in the advancing field of spin electronics.
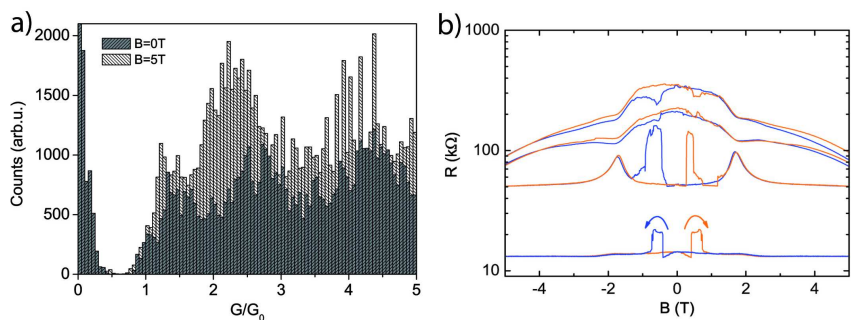

Fig. 2. (a) Comparison of conductance histograms of a Co MCBJ with and without the application of a magnetic field. (b) Typical magnetoresistance traces of Co contacts at different conductance values ranging from $1.3 G_{0}$ to $0.04 G_{0}$.

Figure 2a shows a conductance histogram of a Co MCBJ measured at $4.2 \mathrm{~K}$ with and without the application of a magnetic field. The preferred conductance values are indicated by a broad maximum around $1.3 G_{0}$ and a second one around $2.5 G_{0}$. Usually, a splitting of these maxima would have been expected because of the so-called ballistic magnetoresistance (BMR) [64, 65], where the number of fully transmitted conductance channels changes upon applying a magnetic field. In particular, lifting the spin degeneracy modifies the number of modes for spin-up and spin-down subbands, which manifests itself in a magnetic field dependent opening and closing of discrete conductance channels of the contact leading to discrete conductance steps in the order of $e^{2} / h[66,67]$. Surprisingly, these preferred conductance values did not change noticeably when a magnetic field $(B=5 \mathrm{~T})$ was applied during opening and closing the contact and no preferred conductance at half-integer values of $G_{0}$ are observed. This indicates that the positions of the maxima in the histogram are not a result of the BMR effect. In general, the presence and position of preferred conductance values strongly depends on the contact geometry. In ferromagnetic contacts a diverse behaviour is observed ranging from the complete absence of preferred conductance values or the occurrence of relatively few broad features in the histogram to nearly perfectly quantized conductance in steps of $G_{0} / 2$. The insensitivity of the conductance histogram to the application of a magnetic field is even more astonishing, when one considers the dependence of the junction resistance as a function of magnetic field, the so-called magnetoresistance.

The MR behaviour of the junction can be obtained by monitoring its resistance (or conductance) as the magnetic field is swept from negative to positive values and back again, while keeping the displacement of the electrodes at a fixed position. Few typical examples of MR traces of a Co MCBJ are shown in Fig. 2b for different electrode displacements. These MR traces exhibited a rich behaviour and broad diversity of shapes can be observed, including a GMR-like as well as non-hysteretic and also step-like features.

In order to evaluate these curves, one usually defines the magnetoresistance ratio as $\mathrm{MRR}=\left(R_{\max }-\right.$ $\left.R_{\text {min }}\right) / R_{\text {min }}$. The MR traces in Fig. 2 b show a high MRR of about $70 \%$ in the atomic contact regime. The continuous changes in the contact resistance when applying a magnetic field $B<2 \mathrm{~T}$ are interpreted as a result of reorientation of magnetic domains in the ferromagnetic electrodes. In contrast, for larger fields $(B>2 \mathrm{~T})$ the observed MR effect may be attributed to the enhancement of the saturation field in the constriction. Due to the low coordination of atoms in the constriction, the local band-structure is modified, giving rise to a strong and field-dependent spin-orbit scattering of conduction electron in the contact. This effect, which is known as the atomically enhanced anisotropic magnetoresistance (AAMR), determines both the saturation magnetization and the conductance of the junction. Besides the direct effect of the magnetic field on the transmission of individual conductance channels in the contacts region, the persistence of the GMR-like features indicates that sudden changes in the relative orientation of the magnetization of the electrodes are the main reason for the pronounced MR effect in atomic-sized Co contacts.

Both the atomic configuration in the vicinity of the constriction, giving rise to the AAMR effect, as well as the relative magnetization of the Co electrodes, which manifests itself in the GMR effect, determine the junc- 
tion resistance of in a ferromagnetic MCBJ. Moreover, since the conductance also depends on the electrode displacement, one could argue that magnetoresistive effects may be an additional factor. However, we found that such effect are not strong enough to explain the high MRR observed in the atomic contact regime, while they may be more significant in tunnelling regime [68]. The vacuum tunnelling current depends exponentially on the electrode separation. Hence, a magnetic field induced increase in the electrode separation would lead to an exponential growth of the junction resistance. The distance dependence of the current in the contact regime is weaker than exponential, so that the effect of magnetostriction, although it might be present, is neglectable compared to the large MR effects observed herein.

\section{Potential magnetism in atomic-sized Pt contacts}

Platinum (Pt) is an interesting metal for research as it has quite a few unique properties. In its bulk state it is one of the noble metals, which do not easily corrode like other metals. Nanoparticles of Pt are often used as a catalyst, for instance in catalytic converters in automobiles and in fuel cells, where the size of these nanoparticles is an important parameter [69]. A more recent development in the research of $\mathrm{Pt}$ has been the discovery that it is one of three metals found to form monoatomic chains, along with $\mathrm{Au}$ and Ir [1, 70-73]. During the last decade, theoretical investigations on such atomic-sized Pt contacts have predicted the emergence of magnetism in such small structures [74-76].

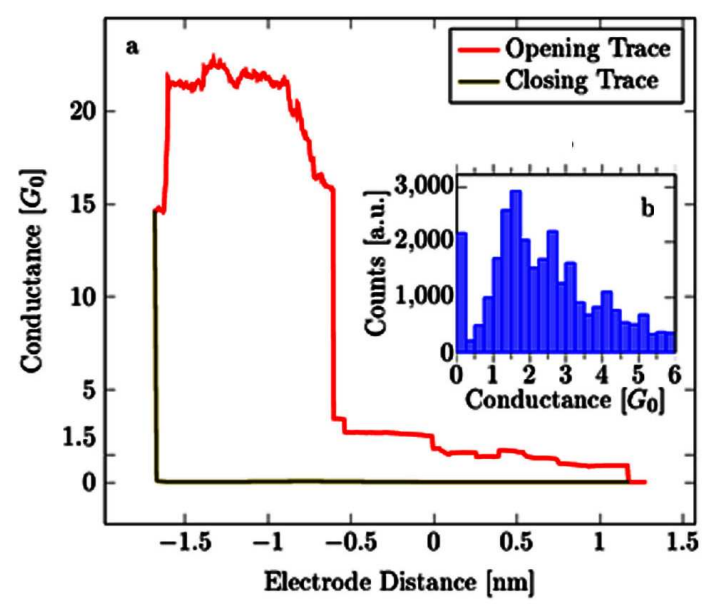

Fig. 3. Typical Pt opening and closing traces; the inset is a histogram constructed from hundreds of opening traces.

However, the experimental investigation of magnetism in Pt, especially in monoatomic chains, has only recently begun. As outlined in Sect. 3, the emergence of magnetism in small Pt contacts may be studied using the MCBJ technique. Figure 3 shows typical opening and closing traces of a Pt MCBJ, from which a conductance histogram (inset of Fig. 3) can be constructed. The chain formation becomes evident from the unusually long plateau of the opening trace at conductance values corresponding to the single-atom contact. Additionally, the chain formation is signified by the large return distance, which is the distance by which the electrode gap must be reduced to reform the metallic contact after fully breaking it.

The histogram in the inset of Fig. 3 shows a broad peak at about $1.6 G_{0}$, corresponding to the expected value for Pt atomic contacts and monoatomic chains. Further peaks are expected at higher values of the conductance, and their strong contribution here can be explained by the fact that our sample are lithographic MCBJs, which are known to have slightly different conductance histograms than MCBJs fabricated with other methods, such as the notched-wire technique using macroscopic wires or even single crystals as a starting material. The higher amount of dislocations and defects in microfabricated MCBJs results in different preferred contact geometries and therefore possibly less pronounced or even shifted maxima in the conductance histograms [77].
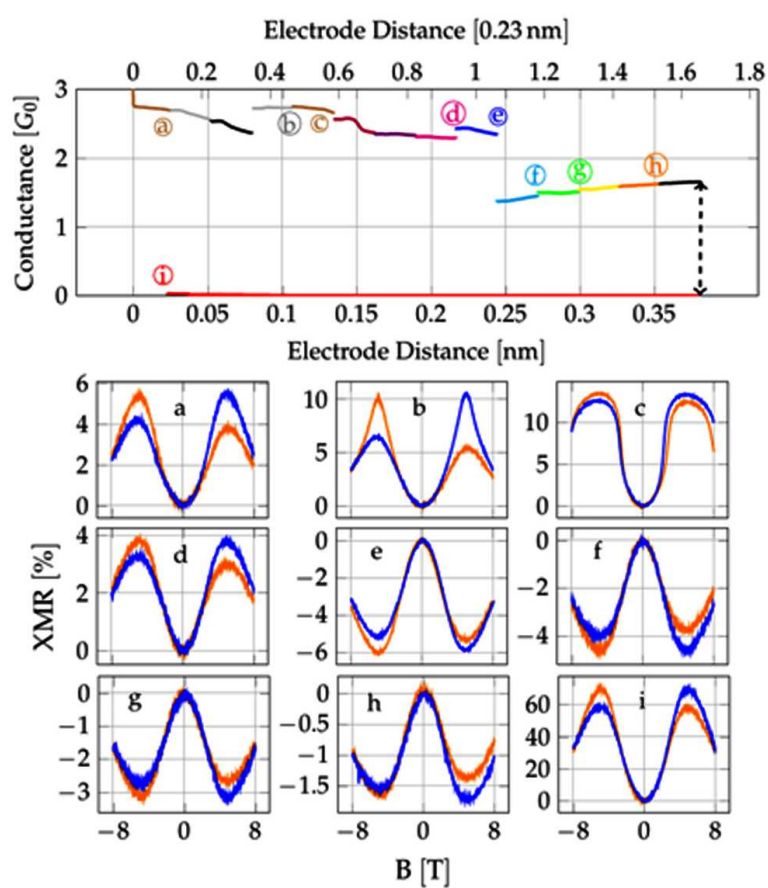

Fig. 4. Selected magnetic field sweep measurements for increasing length of a single monoatomic $\mathrm{Pt}$ chain. Magnetoresistance measurements are performed for different conductance values indicated in the single opening trace (top part). The blue curves represent sweeps from $8 \mathrm{~T}$ to $-8 \mathrm{~T}$ and the orange curves represent sweeps in the opposite direction. Not only does the sign and amplitude of the XMR effect change dramatically, the form also changes.

Magnetoresistance traces are recorded in order to evaluate the effect of external magnetic fields on the conduc- 
tance of the single-atom Pt contact. These measurements are performed while the electrode separation is increased between individual MR sweeps to form a monoatomic $\mathrm{Pt}$ chain. Figure 4 summarizes the results; in part (a) the positions where MR traces are recorded are highlighted in a single opening trace. Below, in part (b), the resulting MR trances are shown. These measurements on $\mathrm{Pt}$ monoatomic contacts show an interesting magnetic field dependence. Firstly, one notices that the magnetoresistive effect (XMR) ranges from as small as one percent (Fig. 4h) to as large as $20 \%$ (Fig. 4c) or even larger across a tunnel junction between two Pt electrodes (Fig. 4i). In addition to this large change in amplitude from contact to contact, one also notes a switching of the sign of the $\mathrm{XMR}$ as well as dramatic changes in the shape of the XMR. Figure 4a shows a continuous modulation of the XRM; Fig. 4b is similar to Fig. 4a, but with an exaggeration of the XMR around $6 \mathrm{~T}$, and the maxima of the $\mathrm{XRM}$ in Fig. 4c are much more rounded with a steeper reduction as the magnetic field approaches $0 \mathrm{~T}$. These effects that can change drastically from contact to contact indicate that the XMR on Pt monoatomic contacts and chains is highly dependent on the exact atomic configuration of the contact. This experimental data goes hand-in-hand with theoretical prediction that even the slightest stretching of the bonds between neighboring atoms can change the magnetic behaviour [71, 74].

Finally, the magnetic field sweeps for each contact show an interesting hysteresis at $6 \mathrm{~T}$ and $-6 \mathrm{~T}$. For example, in Fig. 4b, the down sweep reaches a maximum at about $6 \mathrm{~T}$, continues through its minimum at $0 \mathrm{~T}$ before increasing again. The next local maximum is much smaller than the first maximum reached during the sweep. On the return sweep, the first local maximum is again the largest. The origin of this hysteresis in amplitude is not yet fully clarified.

A possible explanation would be a GMR-like effect that by itself would require a spontaneous magnetization of the two electrodes forming the contacts. However, the macroscopic electrodes consisting of the paramagnet $\mathrm{Pt}$ are known to be non-magnetic as verified by magnetization measurements. Hence, the appearance of this hysteresis might point to magnetically ordered sub-units of the sample on the atomic scale. Both, the observed hysteresis and the high value of the XMR in monoatomic contacts and chains of $\mathrm{Pt}$ strongly suggest that these contacts are indeed magnetic. The high sensitivity of the MR traces to the exact atomic arrangement reveal that the magnetic order, which gives rise to the MR, has to be a local one close to the constriction. Moreover, the local magnetic order also has to depend on atomic-size quantities, such as the atomic orbitals forming the conduction channels. However, recent investigations of the shot-noise of $\mathrm{Pt}$ atomic contacts arranged with the notched-wire MCBJ technique are fully consistent with the assumption of non-spin-polarized transport channels [78]. Therefore the role and emergence of magnetism in such contacts is still uncertain.
In particular, as pointed out in Sect. 3, if the central atom shows a localized magnetic moment, there is reason to believe that the signature of the Kondo effect should be present in the conductance measurements. This issue has attracted considerable interest and different approaches are followed to clarify the open questions. For example, measurements on known reference materials, such as gold may help to understand the unusual MR behaviour observed in Pt contacts. Additionally, modelling the contact geometry [79] and density functional theory (DFT) calculations [80] are performed to determine whether Pt monoatomic chains become magnetic at all.

\section{Heterocontacts based on Au and Co}

The investigations on the homocontacts of Co revealed a very complex behaviour involving effects caused by correlations on the micrometer scale as well as those at the atomic scale. In order to separate the effects of the micromagnetic order from the local ones, we study different magnetic heterocontacts, namely $\mathrm{Au}-\mathrm{Co}-\mathrm{Au}$ and $\mathrm{Co}-\mathrm{Au}-\mathrm{Co}$. Figure 5a shows an example of a $\mathrm{Au}-\mathrm{Co}-\mathrm{Au}$ MCBJ, where a small bridge of Co is deposited between two gold electrodes. Such contacts can be fabricated in a single-step electron beam lithography process by using the shadow evaporation technique to deposit both metals ( $\mathrm{Au}$ and $\mathrm{Co}$ ) onto the same resist mask.
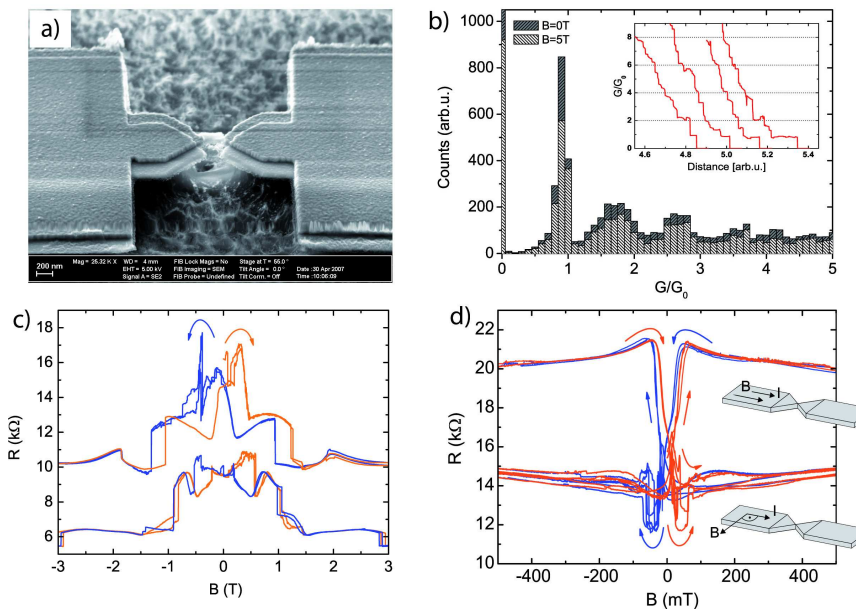

Fig. 5. (a) $\mathrm{Au}-\mathrm{Co}-\mathrm{Au}$ break junction fabricated by means of shadow evaporation. The (brighter) cobalt window in the middle connects to the gold leads. (b) Conductance histogram of the $\mathrm{Au}-\mathrm{Co}-\mathrm{Au}$ sample. A distinct maximum at $1 G_{0}$ can be seen, as well as the typical opening curves for gold break junctions with long conductance plateaus. (c) Typical MR curves for a $\mathrm{Co}-\mathrm{Au}-\mathrm{Co}$ sample in the atomic contact regime with a rich behaviour of different shapes. (d) AMR measured for $\mathrm{Co}-\mathrm{Au}-\mathrm{Co}$ sample for a magnetic field applied in plane, parallel and transversal to the current direction.

For the $\mathrm{Au}-\mathrm{Co}-\mathrm{Au}$ MCBJs, the conductance histogram (Fig. 5b) shows strong similarity to the typical conductance histogram of pristine Au MCBJs, which 
usually exhibit a pronounced and sharp peak at $\approx 1 G_{0}$. Moreover, again there is no significant change of preferred conductance values upon the application of a magnetic field. These findings suggest that, although in principle $\mathrm{Co}-\mathrm{Co}$ and $\mathrm{Co}-\mathrm{Au}$ contacts are possible, in the majority of cases $\mathrm{Au}-\mathrm{Au}$ contacts are formed when breaking the junction.

Interestingly, the $\mathrm{Au}-\mathrm{Co}-\mathrm{Au}$ MCBJs consistently show very low MMR values in the order of $1 \%$, which may indicate that the previously observed MR effects in pristine Co contacts do indeed originate from the contribution of the magnetic electrodes via GMR effects or magnetostriction. In the opposite case of $\mathrm{Co}-\mathrm{Au}-\mathrm{Co}$ MCBJs the conductance histograms suggest that again $\mathrm{Au}-\mathrm{Au}$ contacts are formed preferentially over $\mathrm{Au}-\mathrm{Co}$ and $\mathrm{Co}-\mathrm{Co}$ contacts, although a peak at $1.3 G_{0}$ indicates that $\mathrm{Co}-\mathrm{Co}$ contacts are also formed. However, in the $\mathrm{Au}-\mathrm{Co}-\mathrm{Au}-\mathrm{Co}$ geometry we find much higher MRR values up to $100 \%$.

Figure $5 \mathrm{c}$ also shows that a variety of shapes can be observed in the MR traces, which include rather continuous changes of the resistance but even more pronounced step-like and hysteretic features. The origin of these features is likely to be attributed to the GMR and TMR effect as well as a novel effect resulting in an enhancement of the saturation field in the constriction region. This novel effect, which is due to the increased spin-orbit scattering in the atomic contact regime, leads to a detectable AAMR [81, 82].

The basic mechanism of the AAMR can be summarized as follows: in ultimately small systems, like atomic contacts or chains, the transport channels are determined by the superposition of the atomic orbitals of the atoms forming the contacts and the chain. The conduction channels and their transmissions therefore directly reflect the geometric arrangement of the atoms in the constriction and the local electronic band structure. In macroscopic homogeneous systems, MR effects are small because the high electronic density effectively screens interaction effects resulting, among others, in a quenching of the orbital momentum of the conduction electrons. This screening takes place on a length scale given by the Thomas-Fermi screening length that amounts to only a few nanometres in metals. Hence, for systems smaller than this length scale, the orbital momenta of the conduction electrons are not quenched and may play an important role for the MR. If the size of the external magnetic field is sufficiently large, it aligns the orbitals and thus directly affects the superposition of the wave functions and hence the conduction channels and their transmissions [55, 68]. If this mechanism is the reason for the observed MR, also the effects known from homogeneous systems, such as the AMR, should gradually be enhanced upon decreasing the size of the contacts.

To scrutinize this assumption, we studied the AMR of $\mathrm{Au}-\mathrm{Co}-\mathrm{Au}$ and $\mathrm{Co}-\mathrm{Au}-\mathrm{Co}$ MCBJs in a setup, where the magnetic field is applied in the sample plane both longitudinal and transversal to the current direction, whereas all previous measurements were performed in perpendic- ular fields. An example of the resulting MR traces is shown in Fig. 5d, the schematic insets indicate the relative orientation of current and applied magnetic field. Typically, the $\mathrm{Co}-\mathrm{Au}-\mathrm{Co}$ samples show $\mathrm{AMR}$ values of more than 25\%, while the pristine, unbroken Co MCBJs yield much smaller values of about $2 \%$. These results conclusively demonstrate that the magnitude of the AAMR effect, due to the extreme reduction of the dimensions in atomic size contacts, enhances the influence of an external magnetic field onto the electronic transport.

\section{Summary}

Herein we studied the MR behaviour of magnetic- (Co) and close-to-magnetic $(\mathrm{Pt})$ atomic contacts as well as different types of heterocontacts, such as $\mathrm{Au}-\mathrm{Co}-\mathrm{Au}$ and $\mathrm{Co}-\mathrm{Au}-\mathrm{Co}$, using the mechanically controllable break junction technique. All of these atomic-sized contacts show a rich variety of MR features, which can be attributed to the interplay of different heteroeffects (GMR, TMR) and locally enhanced effects (AAMR) due to the ultimate size-reduction in the constriction region. The later effect could induce magnetic properties at the atomic scale, even in materials that are known to be non-magnetic in the bulk state. This has been demonstrated for Pt contacts, where unusually high MR values of up to $15 \%$ can be observed in the atomic-contact regime. The observation of such high MR effects in homogeneous systems, in particular for materials that are not prone to magnetism, instead of more complex heterostructures, may serve as a principle mechanism for designing novel magneto-electronic devices. However, open questions still include whether a spin-polarisation of the transport channels is either present or absent in such contacts.

\section{Acknowledgments}

We thank C. Bacca, M. Huefner, and M. Suty for their contributions to this work. We are indebted to C. Debuschewitz, H.-F.- Pernau and V. Kunej for help and discussions about cryogenics issues. We profited a lot from discussions with S. Blügel, J.C. Cuevas, M. Häfner, R. Hoffmann, P. Leiderer, H. v. Löhneysen, O. Millo, D. Ralph, C. Sürgers, Y. Yeshurun, and M. Viret. We gratefully acknowledge financial support from the EU FET-open program (STELE FP7-225955), the DFG and the BMBF through a German-Israeli project (DIP), from the Baden-Württemberg-Stiftung through the Research Network Functional Nanostructures, and from the Alfried Krupp von Bohlen and Halbach foundation.

\section{References}

[1] N. Agrait, A.L. Yeyati, J.M. van Ruitenbeek, Phys. Rep. 377, 81 (2003).

[2] A.I. Akimenko, I.K. Yanson, J. Exp. Theor. Phys. 31, 191 (1980). 
[3] J.M. Krans, C.J. Muller, I.K. Yanson, Th.C.M. Govaert, R. Hesper, J.M. van Ruitenbeek, Phys. Rev. B 48, 14721 (1993).

[4] A.G.M. Jansen, A.P. Vangelder, P. Wyder, J. Phys. C, Solid State 13, 6073 (1980).

[5] D. Daghero, R.S. Gonnelli, Supercond. Sci. Tech. 23 043001 (2010).

[6] C.W.J. Beenakker, H. Vanhouten, Phys. Rev. Lett. 66, 3056 (1991).

[7] I.K. Yanson, L.F. Rybalchenko, V.V. Fisun, N.L. Bobrov, V.M. Kirzhner, Yu.D. Tretyakov, A.R. Kaul, I.E. Graboi, Fiz. Nizk. Temp. 14, 732 (1988).

[8] Y.G. Naidyuk, I.K. Yanson, J. Phys., Condens. Matter 10, 8905 (1998).

[9] D. Djukic, J.M. van Ruitenbeek, Nano Lett. 6, 789 (2006).

[10] F. Chen, J. Hihath, Z. Huang, X. Li, N.J. Tao, Annu. Rev. Phys. Chem. 58, 535 (2007).

[11] T. Böhler, A. Edtbauer, E. Scheer, Phys. Rev. B $\mathbf{7 6}$ (2007).

[12] T. Böhler, J. Grebing, A. Mayer-Gindner, H.V. Lohneysen, E. Scheer, Nanotechnology 15, S465 (2004)

[13] Y.R. Chen, L. Zhang, M.S. Hybertsen, Phys. Rev. B 76, 125432 (2007)

[14] J.E. Green, J.W. Choi, A. Boukai, Y. Bunimovich, E. Johnston-Halperin, E. DeIonno, Y. Luo, B.A. Sheriff, K. Xu, Y.S. Shin, H.-R. Tseng, J. Fraser Stoddart, J.R. Heath, Nature 445, 414 (2007).

[15] S. Loth, K. von Bergmann, M. Ternes, A.F. Otte, C.P. Lutz, A.J. Heinrich, Nature Phys. 6, 340 (2010).

[16] M. Mannini, F. Pineider, C. Danieli, F. Totti, L. Sorace, Ph. Sainctavit, M.-A. Arrio, E. Otero, L. Joly, J.C. Cezar, A. Cornia, R. Sessoli, Nature 468, 417 (2010).

[17] M. Mannini, F. Pineider, P. Sainctavit, C. Danieli, E. Otero, C. Sciancalepore, A.M. Talarico, M.-A. Arrio, A. Cornia, D. Gatteschi, R. Sessoli, Nat. Mater. 8, 194 (2009)

[18] P. Gambardella, S. Rusponi, M. Veronese, S.S. Dhesi, C. Grazioli, A. Dallmeyer, I. Cabria, R. Zeller, P.H. Dederichs, K. Kern, C. Carbone, H. Brune, Science 300, 1130 (2003).

[19] C.F. Hirjibehedin, C.-Y. Lin, A.F. Otte, M. Ternes, C.P. Lutz, B.A. Jones, A.J. Heinrich, Science 317, 1199 (2007).

[20] F. Meier, L. Zhou, J. Wiebe, R. Wiesendanger, Science 320, 82 (2008).

[21] A.F. Otte, M. Ternes, K. von Bergmann, S. Loth, H. Brune, C.P. Lutz, C.F. Hirjibehedin, A.J. Heinrich, Nat. Phys. 4, 847 (2008).

[22] H. Brune, P. Gambardella, Surf. Sci. 603, 1812 (2009).

[23] N.I. Bogatina, I.K. Yanson, Zh. Eksp. Teor. Fiz. 63 , $1312(1972)$

[24] I.K. Yanson, Zh. Eksp. Teor. Fiz. 66, 1035 (1974).

[25] I.K. Yanson, A.G. Batrak, J. Exp. Theor. Phys. 27, 197 (1978).

[26] I.K. Yanson, I.O. Kulik, Usp. Fiz. Nauk 128, 364 (1979).
[27] A.G.M. Jansen, F.M. Mueller, P. Wyder, Phys. Rev. B 16, 1325 (1977).

[28] C.J. Muller, J.M. Van Ruitenbeek, L.J. Dejongh, Physica C 191, 485 (1992).

[29] E. Scheer, P. Joyez, M.H. Devoret, D. Esteve, C. Urbina, Superlattices Microstruct. 23, 747 (1998).

[30] E. Scheer, N. Agraït, J.C. Cuevas, A.L. Yeyati, B. Ludoph, A. Martín-Rodero, G.R. Bollinger, J.M. van Ruitenbeek, C. Urbina, Nature 394, 154 (1998).

[31] E. Scheer, P. Joyez, D. Esteve, C. Urbina, M.H. Devoret, Phys. Rev. Lett. 78, 3535 (1997).

[32] C.J. Muller, J.M. van Ruitenbeek, L.J. De Jongh, Phys. Rev. Lett. 69, 140 (1992).

[33] N. Agrait, J.G. Rodrigo, S. Vieira, Phys. Rev. B 47, 12345 (1993).

[34] J.I. Pascual, J. Méndez, J. Gómez-Herrero, A.M. Baró, N. García, V.T. Binh, Phys. Rev. Lett. 71, 1852 (1993).

[35] C. Sirvent, J.G. Rodrigo, N. Agraït, S. Vieira, Physica B 218, 238 (1996).

[36] C. Sirvent, J.G. Rodrigo, S. Vieira, L. Jurczyszyn, N. Mingo, F. Flores, Phys. Rev. B 53, 16086 (1996).

[37] M.A. Reed, C. Zhou, C.J. Muller, T.P. Burgin, J.M. Tour, Science 278, 252 (1997).

[38] M.A. Reed, C. Zhou, M.R. Deshpande, C.J. Muller, T.P. Burgin, L. Jones, II, J.M. Tour, Mol. Electron. Sci. Technol. Acad. Sci. 852, 133 (1998); 852, 133 (1998).

[39] C. Kergueris, J.-P. Bourgoin, S. Palacin, D. Esteve, C. Urbina, M. Magoga, C. Joachim, Phys. Rev. B 59, 12505 (1999).

[40] J.M. van Ruitenbeek, A. Alvarez, I. Piñeyro, C. Grahmann, P. Joyez, M.H. Devoret, D. Esteve, C. Urbina, Rev. Sci. Instrum. 67, 108 (1996).

[41] C. Zhou, C.J. Muller, M.R. Deshpande, J.W. Sleight, M.A. Reed, Appl. Phys. Lett. 67, 1160 (1995).

[42] U. Schröter, E. Scheer, R. Arnold, C. Bacca, T. Böhler, J. Grebing, P. Konrad, V. Kunej, N. Kang, H.-F. Pernau, C. Schirm, Adv. Eng. Mater. 7, 795 (2005).

[43] J.L. Costa-Krämer, Phys. Rev. B 55, R4875 (1997).

[44] F. Elhoussine, S. Mátéfi-Tempfli, A. Encinas, L. Piraux, Appl. Phys. Lett. 81, 1681 (2002).

[45] D.M. Gillingham, I. Linington, C. Müller, J.A.C. Bland, J. Appl. Phys. 93, 7388 (2003).

[46] F. Komori, K. Nakatsuji, Mater. Sci. Eng. B-Solid 84, 102 (2001).

[47] J. Kröger, N. Néel, A. Sperl, Y.F. Wang, and R Berndt, New J. Phys. 11, 125006 (2009).

[48] T. Ono, Y. Ooka, H. Miyajima, Y. Otani, Appl. Phys. Lett. 75, 1622 (1999).

[49] V. Rodrigues, J. Bettini, P.C. Silva, D. Ugarte, Phys. Rev. Lett. 91, 096801 (2003).

[50] M. Shimizu, E. Saitoh, H. Miyajima, Y. Otani, J. Magn. Magn. Mater. 239, 243 (2002).

[51] L. Olesen, E. Laegsgaard, I. Stensgaard, F. Besenbacher, J. Schiotz, P. Stoltze, K.W. Jacobsen, J.K. Norskov, Phys. Rev. Lett. 72, 2251 (1994). 
[52] F. Ott, S. Barberan, J.G. Lunney, J.M.D. Coey, P. Berthet, A.M. de Leon-Guevara, A. Revcolevschi, Phys. Rev. B 58, 4656 (1998).

[53] A. Bagrets, N. Papanikolaou, I. Mertig, Phys. Rev. B 70 (2004).

[54] J.C. Cuevas, A. Levy Yeyati, A. Martín-Rodero, G. Rubio Bollinger, C. Untiedt, N. Agraït, Phys. Rev. Lett. 81, 2990 (1998).

[55] M. Häfner, J.K. Viljas, D. Frustaglia, F. Pauly, M. Dreher, P. Nielaba, J.C. Cuevas, Phys. Rev. B 77, 104409 (2008).

[56] D. Jacob, J. Fernandez-Rossier, J.J. Palacios, Phys. Rev. B 71, 220403(R) (2005).

[57] A.R. Rocha, T. Archer, S. Sanvito, Phys. Rev. B $\mathbf{7 6}$, 220403 (2007).

[58] A. Smogunov, A. Dal Corso, E. Tosatti, Phys. Rev. B 73, 075418 (2006).

[59] J. Velev, W.H. Butler, Phys. Rev. B 69, 094425 (2004).

[60] C. Untiedt, D.M.T. Dekker, D. Djukic, J.M. van Ruitenbeek, Phys. Rev. B 69, 081401 (2004).

[61] M.R. Calvo, J. Fernández-Rossier, J.J. Palacios, D. Jacob, D. Natelson, C. Untiedt, Nature 458, 1150 (2009).

[62] G. Binasch, P. Grünberg, F. Saurenbach, W. Zinn, Phys. Rev. B 39, 4828 (1989).

[63] M.N. Baibich, J.M. Broto, A. Fert, F. Nguyen Van Dau, F. Petroff, P. Etienne, G. Creuzet, A. Friederich, J. Chazelas, Phys. Rev. Lett. 61, 2472 (1988).

[64] H.D. Chopra, S.Z. Hua, Phys. Rev. B 66, 020403 (2002).

[65] N. García, M. Muñoz, G.G. Qian, H. Rohrer, I.G. Saveliev, Y.-W. Zhao, Appl. Phys. Lett. 79, 4550 (2001).

[66] A. Sokolov, C. Zhang, E.Y. Tsymbal, J. Redepenning, B. Doudin, Nat. Nanotechnol. 2, 171 (2007).
[67] H.D. Chopra, M.R. Sullivan, J.N. Armstrong, S.Z. Hua, Nat. Mater. 4, 832 (2005)

[68] S. Egle, C. Bacca, H.-F. Pernau, M. Huefner, D. Hinzke, U. Nowak, E. Scheer, Phys. Rev. B $\mathbf{8 1}$, 134402 (2010).

[69] B. Fan, Y.G. Guo, L.J. Wan, Prog. Chem. 22, 852 (2010).

[70] V. Rodrigues, D. Ugarte, Nanotechnology 13, 404 (2002).

[71] V.M. García-Suárez, A.R. Rocha, S.W. Bailey, C.J. Lambert, S. Sanvito, J. Ferrer, Phys. Rev. Lett. 95, 256804 (2005).

[72] L. Fernandez-Seivane, V.M. Garcia-Suarez, J. Ferrer, Phys. Rev. B 75, 075415 (2007).

[73] A. Thiess, Y. Mokrousov, S. Blug̈el, S. Heinze, Nano Lett. 8, 2144 (2008).

[74] V.M. García-Suárez, D.Zs. Manrique, C.J. Lambert, J. Ferrer, Phys. Rev. B 79, 060408 (2009).

[75] A. Thiess, Y. Mokrousov, S. Heinze, Phys. Rev. B 81, 054433 (2010).

[76] A. Thiess, Y. Mokrousov, S. Heinze, S. Blügel, Phys. Rev. Lett. 103, 217201 (2009)

[77] I.K. Yanson, O.I. Shklyarevskii, J.M. van Ruitenbeek, S. Speller, Phys. Rev. B 77, 033411 (2008).

[78] M. Kumar, R.H.M. Smit, J.M. van Ruitenbeek, O. Tal, arXiv:1101.3939v1 (2011).

[79] F. Pauly, M. Dreher, J.K. Viljas, M. Häfner, J.C. Cuevas, P. Nielaba, Phys. Rev. B 74, 235106 (2006).

[80] A. Delin, E. Tosatti, Surf. Sci. 566, 262 (2004).

[81] B. Hausmanns, T.P. Krome, G. Dumpich, E.F. Wassermann, D. Hinzke, U. Nowak, K.D. Usadel, J. Magn. Magn. Mater. 240, 297 (2002).

[82] M. Viret, M. Gabureac, F. Ott, C. Fermon, C. Barreteau, G. Autes, R. Guirado-Lopez, Eur. Phys. J. B 51, 1 (2006). 